\title{
Distribution of zeros of Dirichlet $L$-functions and the least prime in an arithmetic progression
}

\author{
by
}

JiAnYA LiU (Jinan) and YAngbo Ye (Iowa City, IA)

1. Introduction. The leading term of the $n$-level correlation of nontrivial zeros of an automorphic $L$-function $L(s, \pi)$ for $\mathrm{GL}_{m}$ over $\mathbb{Q}$ is known to be independent of $\pi$, as long as $\pi$ is a cuspidal representation of $\mathrm{GL}_{m}\left(\mathbb{Q}_{\mathbb{A}}\right)$ (Sarnak [13]). This phenomenon is called the universality for the leading term of the $n$-level correlation and was proved for a test function whose Fourier transform has restricted support. In the case of pair $(n=2)$ correlation of nontrivial zeros of Dirichlet $L$-functions $L\left(s, \chi_{1}\right)$ and $L\left(s, \chi_{2}\right)$, we study in this paper behavior of the remainder term of the pair correlation, for the same test function. Our results indicate that the remainder term appears to be either independent of $\chi_{1}$ and $\chi_{2}$, or randomly scattered.

We then conjecture that this phenomenon is also true when the support of the Fourier transform of the test function is enlarged. Under this conjecture and the generalized Riemann hypothesis $(\mathrm{GRH})$, we prove a weighted prime number theorem in arithmetic progressions over a short interval, which in turn implies that the least prime in the arithmetic progression $n \equiv l(\bmod q)$, with $q$ being a prime, $(l, q)=1$, and $l \not \equiv \pm 1(\bmod q)$, is $\ll q^{1+\varepsilon}$. In other words, the Linnik constant is equal to 1 .

Now let us explain our results in a more detailed way. In 1973, Montgomery [11] studied the pair correlation of the zeros of the Riemann zetafunction. Later, Rudnick and Sarnak [12] considered the $n$-level correlation of nontrivial zeros of principal $L$-functions $L(s, \pi)$ attached to an automorphic irreducible cuspidal representation $\pi$ of $\mathrm{GL}_{m}$ over $\mathbb{Q}$. To state the result of [12] in the special case of $m=1$ and $n=2$, we let $\chi_{j}$ be a primitive character modulo $q$, and $L\left(s, \chi_{j}\right)$ the corresponding Dirichlet $L$-function, for

2000 Mathematics Subject Classification: 11M26, $11 \mathrm{~N} 13$.

The first-named author supported in part by China NNSF grant \# 10125101, and by a Ministry of Education Major Grant Program in Sciences and Technology.

The second-named author supported in part by the USA NSA under grant number MDA904-03-1-0066. 
$j=1,2$. For

$$
g_{j} \in C_{\mathrm{c}}^{\infty}(\mathbb{R}) \quad \text { with } \operatorname{supp}\left(g_{j}\right)=[-a, a]
$$

we set

$$
h_{j}(r)=\int_{\mathbb{R}} g_{j}(v) e^{i v r} d v,
$$

and $\mathbf{h}=\left(h_{1}, h_{2}\right)$. Let $\Phi \in C_{\mathrm{c}}^{1}(\mathbb{R})$, and

$$
f\left(x_{1}, x_{2}\right)=\int_{\mathbb{R}} e\left(-\left(x_{1}-x_{2}\right) v\right) \Phi(v) d v,
$$

where $e(t)=e^{2 \pi i t}$ as usual. Consider

$$
F\left(\mathbf{h}, T, \Phi ; \chi_{1}, \chi_{2}\right)=\sum_{\gamma_{1}, \gamma_{2}} h_{1}\left(\frac{\gamma_{1}}{T}\right) h_{2}\left(\frac{\gamma_{2}}{T}\right) f\left(\frac{L}{2 \pi} \gamma_{1}, \frac{L}{2 \pi} \gamma_{2}\right)
$$

with $L=\log T$, where $\varrho_{j}=1 / 2+i \gamma_{j}$ is taken over nontrivial zeros of $L\left(s, \chi_{j}\right)$. This function measures, as $T \rightarrow \infty$, the pair correlation of nontrivial zeros of $L(s, \chi)$ if $\chi_{1}=\chi_{2}=\chi$, and the pair correlation between nontrivial zeros of $L\left(s, \chi_{1}\right)$ and $L\left(s, \chi_{2}\right)$ if $\chi_{1} \neq \chi_{2}$.

Rudnick and Sarnak [12] proved that if $\chi_{1}=\chi_{2}=\chi$ and $\operatorname{supp}(\Phi) \subset$ $(-1,1)$, then

$$
F(\mathbf{h}, T, \Phi ; \chi, \chi)=\frac{\kappa(\mathbf{h})}{2 \pi} T L\left(\Phi(0)+\int_{\mathbb{R}}|v| \Phi(v) d v\right)+O(T),
$$

where

$$
\kappa(\mathbf{h})=\int_{\mathbb{R}} h_{1}(r) h_{2}(r) d r .
$$

Another version of this was proved for the Riemann zeta function in Liu and Ye [10].

Recently the authors [9] studied the $n$-level correlation of nontrivial zeros of distinct $L$-functions attached to automorphic irreducible cuspidal representations of $\mathrm{GL}_{m}$ over $\mathbb{Q}$. In the special case of $m=1$ and $n=2$, our result asserts that, when $\chi_{1} \neq \chi_{2}$,

$$
F\left(\mathbf{h}, T, \Phi ; \chi_{1}, \chi_{2}\right)=\frac{\kappa(\mathbf{h})}{2 \pi} \Phi(0) T L+O(T)
$$

for any function $\Phi$ with support $\subset(-1,1)$. Note that the results $(1.4)$ and (1.6) do not depend on GRH.

We observe that the main terms in (1.4) and (1.6) are independent of the character $\chi$ and characters $\chi_{1}$ and $\chi_{2}$, respectively. For automorphic $L$-functions attached to cuspidal representations of $\mathrm{GL}_{m}$ over $\mathbb{Q}$, this was first discovered by Rudnick and Sarnak [12], and is known as the universality of the distribution of nontrivial zeros. 
It is believed that the pair correlation and hence the universality of zeros should have applications in distribution of primes. We realized, however, that we actually need not only this universality for the leading terms of $F\left(\mathbf{h}, T, \Phi ; \chi_{1}, \chi_{2}\right)$ in (1.4) and (1.6), but also information on the remainder terms.

The first purpose of this paper is thus to study nontrivial zeros for the remainder terms. From now on, $\chi_{j}$ is a Dirichlet character modulo $q$ not necessarily primitive.

Theorem 1.1. Assume $\operatorname{supp}(\Phi)=\left[b_{1}, b_{2}\right] \subset(1 / 2,1)$. For a prime number $q$ with $T^{1-b_{1}} \leq q \leq T^{b_{1}-\varepsilon}$, let $l \not \equiv \pm 1(\bmod q)$ with $(l, q)=1$. Then

$$
\sum_{\chi_{1}, \chi_{2} \bmod q} \chi_{1}(l) \bar{\chi}_{2}(l) F\left(\mathbf{h}, T, \Phi ; \chi_{1}, \chi_{2}\right) \ll \varphi(q) T L
$$

where the implied constant depends on $\Phi, b_{1}, b_{2}, g_{1}$, and $g_{2}$ only.

We remark that the mean value estimate in (1.7) cannot be obtained using the individual bounds in (1.4) and (1.6). Indeed, as we assume that $\Phi(0)=0$, the main term in (1.6) and a part of the main term in (1.4) disappear. The remaining part of the main term in (1.4) yields $O(\varphi(q) T L)$. But the sum of $O(T)$ over $\chi_{1}, \chi_{2} \bmod q$ would give us a bigger $\varphi^{2}(q) T$. In other words, a factor $\varphi(q) / L$ is saved in (1.7). This saving represents cancellation among the remainder terms multiplied by $\chi_{1}(l) \bar{\chi}_{2}(l)$ over $\chi_{1}$ and $\chi_{2}$. This can be interpreted as a manifestation of either the universality for the remainder terms, or the fact that the remainder terms might be randomly scattered. In applications, it is often crucial to consider big $q$ compared with $T$. In this case, the above saving becomes crucial.

In Theorem 1.1, we assume $q$ to be a prime with $l \not \equiv \pm 1(\bmod q)$; see Lemmas 3.1 and 3.2. Indeed, when $l \equiv 1(\bmod q)$, we have $\chi_{1}(l) \bar{\chi}_{2}(l)=1$ and cannot control the size of the left side of (1.7). More specifically, Lemma 3.2 is not valid for $l \equiv \pm 1(\bmod q)$.

We believe that Theorem 1.1 is still true without the restriction on the support of $\Phi$.

ConjeCture 1.2. Let $q$ and $l$ be as in Theorem 1.1. Then Theorem 1.1 holds for $\Phi$ with $\operatorname{supp}(\Phi)=\left[b_{1}, b_{2}\right] \subset(1 / 2, \infty)$.

Conjecture 1.2 has applications to classical problems in distribution of primes, for example Linnik's constant. In view of Dirichlet's theorem that there are infinitely many primes in the arithmetic progression $n \equiv l(\bmod q)$ with $(q, l)=1$, it is a natural question how big is the least prime, denoted by $P(q, l)$, in this arithmetic progression. Linnik [7]-[8] proved that there is an absolute constant $\ell>0$ such that $P(q, l) \ll q^{\ell+\varepsilon}$, and this constant $\ell$ was named after him. Since then, a number of authors have established numerical 
values for Linnik's constant $\ell$; the best result known is $\ell=5.5$ by HeathBrown [4]. We remark that these results depend on, among other things, numerical estimates concerning zero-free regions and the Deuring-Heilbronn phenomenon of Dirichlet $L$-functions. Under GRH the above bounds can be improved to

$$
P(q, l) \ll \varphi^{2}(q) \log ^{2} q .
$$

The second purpose of this paper is to sharpen the estimate in (1.8) under GRH and Conjecture 1.2.

Theorem 1.3. Assume GRH and Conjecture 1.2. Let $q$ be a prime with $l \not \equiv \pm 1(\bmod q)$ and $(l, q)=1$. Then for arbitrary $\varepsilon>0$,

$$
P(q, l) \ll_{\varepsilon} q^{1+\varepsilon} .
$$

This bound is the best possible save the $\varepsilon$ in the exponent. In fact, a trivial lower bound for $P(q, l)$ is

$$
\max _{l} P(q, l) \geq(1+o(1)) \varphi(q) \log q .
$$

Granville and Pomerance [2] proved an improvement to this. Theorem 1.3 will be derived from a weighted prime number theorem (Theorem 7.2).

Assuming GRH and a conjecture on the rate of convergence of the (1-level) linear density of low-lying zeros of Dirichlet $L$-functions, Sarnak [14] proved the same bound for the least prime in an arithmetic progression. The conjecture he used is indeed a conjecture on the size of the remainder terms, while our Conjecture 1.2 predicts not the size but mean value of the remainder terms.

Hughes and Rudnick [5] computed unweighted 1-level statistics of lowlying zeros of Dirichlet $L$-functions.

Languasco and Perelli [6] considered sums of the pair correlation of nontrivial zeros of Dirichlet $L$-functions with weights of Gauss sums $\tau(\chi)$ multiplied by characters

$$
\sum_{\chi_{1}, \chi_{2} \bmod q} \chi_{1}(l) \bar{\chi}_{2}(l) \tau\left(\bar{\chi}_{1}\right) \tau\left(\chi_{2}\right) F\left(\mathbf{h}, T, \Phi ; \chi_{1}, \chi_{2}\right) .
$$

Using this estimation, they studied the exceptional set in the Goldbach problem. Without Gauss sums in the weights, our sum in Theorem 1.1 might have different flexibility in possible applications.

The authors would like to thank Peter Sarnak for helpful, detailed comments, and for showing us his approach in [14] to the problem, and to thank the referee for helpful suggestions, especially for his simple proof of Lemma 3.2 as is shown in the paper. 
2. $L$-functions. Let $\chi^{*} \bmod q^{*}$ be the primitive character inducing the given character $\chi \bmod q$, with $\operatorname{cond}(\chi)=q^{*}$. Then (see e.g. [1, Chap. 5, (3)])

$$
L(s, \chi)=L\left(s, \chi^{*}\right) \prod_{\substack{p \mid q \\ p \nmid q^{*}}}\left(1-\frac{\chi^{*}(p)}{p^{s}}\right)=: L\left(s, \chi^{*}\right) M(s, \chi),
$$

say. The following explicit formula is a generalization of that of Rudnick and Sarnak; see [12, Proposition 2.1].

Lemma 2.1. Let $g, h$ be defined as in (1.1) and (1.2) respectively. Let $\chi \bmod q$ be a Dirichlet character induced by $\chi^{*} \bmod q^{*}$, and $\varrho=1 / 2+i \gamma$ be zeros of $L(s, \chi)$ within $0<\operatorname{Re}(s)<1$. Then

$$
\begin{aligned}
\sum_{\gamma} h(\gamma)= & \delta_{\chi}\left\{h\left(-\frac{i}{2}\right)+h\left(\frac{i}{2}\right)\right\}+\frac{1}{2 \pi} \int_{\mathbb{R}} h(r) \Omega(r, \chi) d r \\
& -\sum_{n=1}^{\infty}\left(\frac{\Lambda(n) \chi(n)}{\sqrt{n}} g(\log n)+\frac{\Lambda(n) \bar{\chi}(n)}{\sqrt{n}} g(-\log n)\right),
\end{aligned}
$$

where $\delta_{\chi}=1$ or 0 according as $\chi=\chi^{0} \bmod q$ or not, and

$$
\begin{aligned}
\Omega(x, \chi)= & \log \operatorname{cond}(\chi)+\frac{\Gamma_{\mathbb{R}}^{\prime}}{\Gamma_{\mathbb{R}}}\left(\frac{1}{2}+\mu(\chi)+i x\right) \\
& +\frac{\Gamma_{\mathbb{R}}^{\prime}}{\Gamma_{\mathbb{R}}}\left(\frac{1}{2}+\mu(\chi)-i x\right) \\
& -\frac{M^{\prime}}{M}\left(\frac{1}{2}+i x, \chi\right)-\frac{M^{\prime}}{M}\left(\frac{1}{2}-i x, \bar{\chi}\right),
\end{aligned}
$$

with $\Gamma_{\mathbb{R}}(s)=\pi^{-s / 2} \Gamma(s / 2), \mu(\chi)=0$ if $\chi(-1)=1, \mu(\chi)=1$ if $\chi(-1)=-1$.

Proof. Since $\chi^{*}$ is primitive, the functional equation of $L\left(s, \chi^{*}\right)$ takes the form

$$
\Phi\left(s, \chi^{*}\right)=\varepsilon\left(s, \chi^{*}\right) \Phi\left(1-s, \bar{\chi}^{*}\right),
$$

where

$$
\Phi\left(s, \chi^{*}\right)=\Gamma_{\mathbb{R}}\left(s+\mu\left(\chi^{*}\right)\right) L\left(s, \chi^{*}\right) .
$$

The $\varepsilon$ factor $\varepsilon\left(s, \chi^{*}\right)$ satisfies $\varepsilon\left(s, \chi^{*}\right)=\tau(\chi)\left(q^{*}\right)^{-s}$.

Let $H(s)=h((s-1 / 2) / i)$, and consider the integral

$$
J=\frac{1}{2 \pi i} \int_{\operatorname{Re}(s)=2} \frac{\Phi^{\prime}}{\Phi}\left(s, \chi^{*}\right) H(s) d s .
$$

Since $|H(s)|$ is rapidly decreasing in $|\operatorname{Im}(s)|$ and is entire, the integral $J$ converges absolutely, and all the contour shifts below are legitimate. Obviously $\left(\Phi^{\prime} / \Phi\right)\left(s, \chi^{*}\right)$ has simple poles at the zeros of $\Phi\left(s, \chi^{*}\right)$ with residues the multiplicity of the zero. In the case of $\Phi\left(s, \chi^{*}\right)=\zeta(s),\left(\Phi^{\prime} / \Phi\right)\left(s, \chi^{*}\right)$ has 
a simple pole with residue -1 at the poles $s=0,1$. Shifting the contour in (2.5) to $\operatorname{Re}(s)=-1$, we have

$$
J=-\delta_{\chi^{*}}\left\{h\left(-\frac{i}{2}\right)+h\left(\frac{i}{2}\right)\right\}+\sum_{\gamma} h(\gamma)+\frac{1}{2 \pi i} \int_{\operatorname{Re}(s)=-1} \frac{\Phi^{\prime}}{\Phi}\left(s, \chi^{*}\right) H(s) d s
$$

where the sum is over the nontrivial zeros of $L\left(s, \chi^{*}\right)$, each counted with its multiplicity, and $\delta_{\chi^{*}}=1$ or 0 according as $\chi^{*}=\chi^{0} \bmod 1$ (i.e. $L\left(s, \chi^{*}\right)=$ $\zeta(s))$ or not. The functional equation (2.4) now gives

$$
\frac{\Phi^{\prime}}{\Phi}\left(s, \chi^{*}\right)=-\log q^{*}-\frac{\Phi^{\prime}}{\Phi}\left(1-s, \bar{\chi}^{*}\right) .
$$

Using this and changing variables, we get

$$
\begin{aligned}
J= & -\delta_{\chi^{*}}\left\{h\left(-\frac{i}{2}\right)+h\left(\frac{i}{2}\right)\right\}+\sum_{\gamma} h(\gamma)-\frac{1}{2 \pi i} \int_{\operatorname{Re}(s)=2} H(s) \log q^{*} d s \\
& -\frac{1}{2 \pi i} \int_{\operatorname{Re}(s)=2} \frac{\Phi^{\prime}}{\Phi}\left(s, \chi^{*}\right) H(1-s) d s .
\end{aligned}
$$

Consequently,

$$
\begin{aligned}
\sum_{\gamma} h(\gamma)= & \delta_{\chi^{*}}\left\{h\left(-\frac{i}{2}\right)+h\left(\frac{i}{2}\right)\right\}+\frac{1}{2 \pi i} \int_{\operatorname{Re}(s)=2} H(s) \log q^{*} d s \\
& +\frac{1}{2 \pi i} \int_{\operatorname{Re}(s)=2} \frac{\Phi^{\prime}}{\Phi}\left(s, \chi^{*}\right) H(s) d s \\
& +\frac{1}{2 \pi i} \int_{\operatorname{Re}(s)=2} \frac{\Phi^{\prime}}{\Phi}\left(s, \bar{\chi}^{*}\right) H(1-s) d s .
\end{aligned}
$$

By (2.1) and the definition of $\Phi\left(s, \chi^{*}\right)$,

$$
\begin{aligned}
& \frac{1}{2 \pi i} \int_{\operatorname{Re}(s)=2} \frac{\Phi^{\prime}}{\Phi}\left(s, \chi^{*}\right) H(s) d s \\
& \quad=\frac{1}{2 \pi i} \int_{\operatorname{Re}(s)=2}\left\{\frac{\Gamma_{\mathbb{R}}^{\prime}}{\Gamma_{\mathbb{R}}}\left(s+\mu\left(\chi^{*}\right)\right)+\frac{L^{\prime}}{L}(s, \chi)-\frac{M^{\prime}}{M}(s, \chi)\right\} H(s) d s .
\end{aligned}
$$

Now shifting the contour to $\operatorname{Re}(s)=1 / 2$,

$$
\begin{aligned}
& \frac{1}{2 \pi i} \int_{\operatorname{Re}(s)=2}\left\{\frac{\Gamma_{\mathbb{R}}^{\prime}}{\Gamma_{\mathbb{R}}}\left(s+\mu\left(\chi^{*}\right)\right)-\frac{M^{\prime}}{M}(s, \chi)\right\} H(s) d s \\
&=\frac{1}{2 \pi} \int_{\mathbb{R}}\left\{\frac{\Gamma_{\mathbb{R}}^{\prime}}{\Gamma_{\mathbb{R}}}\left(s+\mu\left(\chi^{*}\right)\right)-\frac{M^{\prime}}{M}(s, \chi)\right\} h(r) d r,
\end{aligned}
$$


where $s=1 / 2+i r$. For $\operatorname{Re}(s)>1$, we have

$$
\frac{L^{\prime}}{L}(s, \chi)=-\sum_{n=1}^{\infty} \frac{\Lambda(n) \chi(n)}{n^{s}},
$$

and consequently,

$$
\begin{aligned}
\frac{1}{2 \pi i} \int_{\operatorname{Re}(s)=2} \frac{L^{\prime}}{L}(s, \chi) H(s) d s & =-\frac{1}{2 \pi} \sum_{n=1}^{\infty} \frac{\Lambda(n) \chi(n)}{\sqrt{n}} \int_{\mathbb{R}} h(r) e^{-i r \log n} d r \\
& =-\sum_{n=1}^{\infty} \frac{\Lambda(n) \chi(n)}{\sqrt{n}} g(\log n) .
\end{aligned}
$$

We do the same for the integral involving $H(1-s)$. Collecting all of these into (2.6), we obtain

$$
\begin{aligned}
\sum_{\gamma} h(\gamma)= & \delta_{\chi^{*}}\left\{h\left(-\frac{i}{2}\right)+h\left(\frac{i}{2}\right)\right\}+\frac{1}{2 \pi} \int_{\mathbb{R}} h(r)\left\{\log q^{*}\right. \\
& +\frac{\Gamma_{\mathbb{R}}^{\prime}}{\Gamma_{\mathbb{R}}}\left(\frac{1}{2}+\mu\left(\chi^{*}\right)+i r\right)+\frac{\Gamma_{\mathbb{R}}^{\prime}}{\Gamma_{\mathbb{R}}}\left(\frac{1}{2}+\mu\left(\chi^{*}\right)-i r\right) \\
& \left.-\frac{M^{\prime}}{M}\left(\frac{1}{2}+i r, \chi\right)-\frac{M^{\prime}}{M}\left(\frac{1}{2}-i r, \bar{\chi}\right)\right\} d r \\
& -\sum_{n=1}^{\infty}\left(\frac{\Lambda(n) \chi(n)}{\sqrt{n}} g(\log n)+\frac{\Lambda(n) \bar{\chi}(n)}{\sqrt{n}} g(-\log n)\right) .
\end{aligned}
$$

This gives (2.2) on noting that $\delta_{\chi}=\delta_{\chi^{*}}, \mu(\chi)=\mu\left(\chi^{*}\right)$, and that the nontrivial zeros of $L\left(s, \chi^{*}\right)$ are just those of $L(s, \chi)$ within $0<\operatorname{Re}(s)<1$.

With the test functions

$$
H_{T}(r)=h(r / T) e^{-i L r u}, \quad G_{T}(v)=T g(T(L u+v)),
$$

the above explicit formula takes the following form.

LEMMA 2.2. Under the same condition of Lemma 2.1, we have

$$
\begin{aligned}
\sum_{\gamma} h\left(\frac{\gamma}{T}\right) e^{-i L \gamma u}= & \delta_{\chi} P(T, u)+T g_{T}(T L u, \chi) \\
& +T S^{+}(T, u, \chi)+T S^{-}(T, u, \bar{\chi})
\end{aligned}
$$

where

$$
\begin{aligned}
P(T, u) & =h\left(-\frac{i}{2 T}\right) T^{-u / 2}+h\left(\frac{i}{2 T}\right) T^{u / 2}, \\
S^{ \pm}(T, u, \chi) & =-\sum_{n=1}^{\infty} \frac{\Lambda(n) \chi(n)}{\sqrt{n}} g(T(L u \pm \log n)),
\end{aligned}
$$




$$
g_{T}(x, \chi)=\frac{1}{2 \pi} \int_{\mathbb{R}} h(r) \Omega(r T, \chi) e^{-i r x} d r
$$

with $\Omega(x, \chi)$ as in $(2.3)$.

The above formula will be expressed in a form suitable for our later applications. To this end, we consider the term

$$
\begin{aligned}
h\left(\frac{i}{2 T}\right) T^{u / 2} & =T^{u / 2} \int_{\mathbb{R}} g(y) e^{-y /(2 T)} d y \\
& =T^{u / 2}\left(\int_{-\infty}^{T L u}+\int_{T L u}^{\infty}\right) g(y) e^{-y /(2 T)} d y .
\end{aligned}
$$

We change variables to $v$ by $y=T(L u-\log v)$ in the first integral and by $y=T(L u+\log v)$ in the second integral for $1 \leq v<\infty$. Then

$$
\begin{aligned}
h\left(\frac{i}{2 T}\right) T^{u / 2}= & T^{u / 2} \int_{1}^{\infty} g(T(L u-\log v)) e^{-(L u-\log v) / 2} \frac{T d v}{v} \\
& +T^{u / 2} \int_{1}^{\infty} g(T(L u+\log v)) e^{-(L u+\log v) / 2} \frac{T d v}{v} \\
= & T \int_{1}^{\infty} g(T(L u-\log v)) \frac{d v}{\sqrt{v}}+T \int_{1}^{\infty} g(T(L u+\log v)) \frac{d v}{v^{3 / 2}}
\end{aligned}
$$

Similarly,

$$
h\left(-\frac{i}{2 T}\right) T^{-u / 2}=T \int_{1}^{\infty} g(T(L u+\log v)) \frac{d v}{\sqrt{v}}+T \int_{1}^{\infty} g(T(L u-\log v)) \frac{d v}{v^{3 / 2}} .
$$

Consequently,

$$
P(T, u)=T \int_{1}^{\infty}\{g(T(L u-\log v))+g(T(L u+\log v))\}\left(\frac{1}{\sqrt{v}}+\frac{1}{v^{3 / 2}}\right) d v .
$$

Now we split the above integral into two parts using $d v=d[v]+d(v-[v])$, and get

$$
\begin{aligned}
P(T, u)= & T \sum_{n=1}^{\infty}\left(\frac{1}{\sqrt{n}}+\frac{1}{n^{3 / 2}}\right) \\
& \times\{g(T(L u-\log n))+g(T(L u+\log n))\} \\
& +T \int_{1}^{\infty}\{g(T(L u-\log v))+g(T(L u+\log v))\} \\
& \times\left(\frac{1}{\sqrt{v}}+\frac{1}{v^{3 / 2}}\right) d(v-[v]) .
\end{aligned}
$$


Let

$$
J^{ \pm}(T, u)=\int_{1}^{\infty} g(T(L u \pm \log v))\left(\frac{1}{\sqrt{v}}+\frac{1}{v^{3 / 2}}\right) d(v-[v]) .
$$

Inserting (2.11) and (2.12) into (2.7), we get the following result.

LEMMA 2.3. Under the assumption of Lemma 2.1, we have, for any $u$,

$$
\begin{aligned}
\sum_{\gamma} h\left(\frac{\gamma}{T}\right) e^{-i L \gamma u}= & T g_{T}(T L u, \chi)+T Z^{+}(T, u, \chi) \\
& +T Z^{-}(T, u, \bar{\chi})+\delta_{\chi} T J^{+}(T, u)+\delta_{\chi} T J^{-}(T, u),
\end{aligned}
$$

where $J^{ \pm}(T, u)$ are given as in $(2.12)$, and

$$
Z^{ \pm}(T, u, \chi)=-\sum_{n=1}^{\infty} w(n, \chi) g(T(L u \pm \log n)),
$$

with

$$
w(n, \chi)=\frac{\Lambda(n) \chi(n)}{\sqrt{n}}-\delta_{\chi}\left(\frac{1}{\sqrt{n}}+\frac{1}{n^{3 / 2}}\right) .
$$

3. Mean value of $g_{T}(x, \chi)$ over $\chi$. The following mean-value estimate for $g_{T}(x, \chi)$ is crucial in our later argument.

Lemma 3.1. Let $q \geq 5$ be a prime and $l \not \equiv \pm 1(\bmod q)$. Then

$$
\begin{aligned}
& \sum_{\chi \bmod q} \chi(l) g_{T}(x, \chi) \ll e^{-\sigma|x|} \log q+e^{-|x| /(2 T)}, \\
& \sum_{\chi \bmod q} \bar{\chi}(l) g_{T}(x, \chi) \ll e^{-\sigma|x|} \log q+e^{-|x| /(2 T)},
\end{aligned}
$$

where $\sigma \geq 1$ is any positive number.

The proof of Lemma 3.1 depends on the following lemma.

Lemma 3.2. Let $q \geq 5$ and $l \not \equiv \pm 1(\bmod q)$. Then

$$
\sum_{\substack{\chi \bmod q \\ \chi(-1)=1}} \chi(l)=\sum_{\substack{\chi \bmod q \\ \chi(-1)=-1}} \chi(l)=0 .
$$

Proof. We have $\chi(-1)=1$ if and only if $\chi$ is the square of another character, $\psi^{2}$ say, and each even character arises exactly twice in this way. Hence the first sum is

$$
\frac{1}{2} \sum_{\psi \bmod q} \psi^{2}(l)=\frac{1}{2} \sum_{\psi \bmod q} \psi\left(l^{2}\right)=0
$$


since $l^{2} \not \equiv 1(\bmod q)$. Using $l \not \equiv 1(\bmod q)$ again, we have

$$
0=\sum_{\chi \bmod q} \chi(l)=\sum_{\substack{\chi \bmod q \\ \chi(-1)=1}} \chi(l)+\sum_{\substack{\chi \bmod q \\ \chi(-1)=-1}} \chi(l) .
$$

This proves that the second sum is also zero.

Proof of Lemma 3.1. We will only prove (3.1) since the proof of (3.2) is exactly the same. Without loss of generality, we can suppose $x \geq 0$. Rewrite (2.10) as

$$
\begin{aligned}
g_{T}(x, \chi) & =\frac{1}{2 \pi i} \int_{\operatorname{Re}(s)=0} h\left(\frac{s}{i}\right) \Omega\left(\frac{s T}{i}, \chi\right) e^{-s x} d s \\
& =\frac{1}{2 \pi i} \int_{\operatorname{Re}(s)=0} h\left(\frac{s}{i}\right) \omega(s, \chi) e^{-s x} d s,
\end{aligned}
$$

where

$$
\begin{aligned}
\omega(s, \chi)= & \log \operatorname{cond}(\chi)+\frac{\Gamma_{\mathbb{R}}^{\prime}}{\Gamma_{\mathbb{R}}}\left(\frac{1}{2}+\mu(\chi)+s T\right) \\
& +\frac{\Gamma_{\mathbb{R}}^{\prime}}{\Gamma_{\mathbb{R}}}\left(\frac{1}{2}+\mu(\chi)-s T\right)-\frac{M^{\prime}}{M}\left(\frac{1}{2}+s T, \chi\right) \\
& -\frac{M^{\prime}}{M}\left(\frac{1}{2}-s T, \bar{\chi}\right) .
\end{aligned}
$$

By Stirling's formula and the definition of $M(s, \chi)$, we have

$$
\omega(s, \chi) \ll \log (q T|s|)
$$

for any $s$ in a vertical strip $|\operatorname{Re}(s)| \leq B$ with $|s|$ sufficiently large $(s$ is not necessarily on the line $\operatorname{Re}(s)=0)$. Also $|h(s)|$ is rapidly decreasing as $|\operatorname{Re}(s)| \rightarrow \infty$. Therefore we can shift the contour of integration to the line $\operatorname{Re}(s)=\sigma$ where $\sigma$ is any positive number. This gives

$$
g_{T}(x, \chi)=\frac{1}{2 \pi i} \int_{\operatorname{Re}(s)=\sigma} h\left(\frac{s}{i}\right) \omega(s, \chi) e^{-s x} d s-R(x, \chi)
$$

where $R(x, \chi)$ is the sum of the residues at the poles passed when shifting the contour.

We now compute $R(x, \chi)$. Since $\mu(\chi) \geq 0$, the first $\Gamma$ term in (3.3) is holomorphic in $\operatorname{Re}(s) \geq 0$. The second $\Gamma$ term has simple poles at

$$
s_{k}(\chi)=\frac{1 / 2+\mu(\chi)+2 k}{T}, \quad 0 \leq k<\frac{1}{2}\left(\sigma T-\frac{1}{2}-\mu(\chi)\right)
$$


with residues

$$
\frac{1}{T} \sum_{0 \leq k<(\sigma T-1 / 2-\mu(\chi)) / 2} h\left(\frac{s_{k}(\chi)}{i}\right) \exp \left(-s_{k}(\chi) x\right) .
$$

Since $q \geq 5$ is prime, we deduce from $(2.1)$ that $L(s, \chi)=L\left(s, \chi^{*}\right)$ for any nonprincipal character $\chi \bmod q$, and that $L\left(s, \chi^{0}\right)=\zeta(s)\left(1-q^{-s}\right)$. Therefore,

$$
M(s, \chi)= \begin{cases}1-q^{-s} & \text { if } \chi=\chi^{0} \bmod q \\ 1 & \text { otherwise. }\end{cases}
$$

Hence in (3.3), the $M$ terms appear only when $\chi$ is principal, and

$$
\frac{M^{\prime}}{M}\left(\frac{1}{2} \pm s T, \chi^{0}\right)=\frac{\log q}{q^{1 / 2 \pm s T}-1} .
$$

The first $M$ term in (3.3) is holomorphic in $\operatorname{Re}(s) \geq 0$. The second one has simple poles at

$$
\varpi_{m}=\frac{1}{2 T}+\frac{2 \pi i m}{\log q}, \quad m \in \mathbb{Z},
$$

with residues

$$
h\left(\frac{\varpi_{m}}{i}\right) \exp \left(-\varpi_{m} x\right)
$$

Consequently,

$$
\begin{aligned}
R(x, \chi)= & \frac{1}{T} \sum_{0 \leq k \leq \sigma T / 2} h\left(\frac{s_{k}(\chi)}{i}\right) \exp \left(-s_{k}(\chi) x\right) \\
& +\delta_{\chi} \sum_{m \in \mathbb{Z}} h\left(\frac{\varpi_{m}}{i}\right) \exp \left(-\varpi_{m} x\right) .
\end{aligned}
$$

Summing (3.5) over $\chi$, we get

$$
\begin{aligned}
\sum_{\chi \bmod q} \chi(l) g_{T}(x, \chi)= & \frac{1}{2 \pi i} \int_{\operatorname{Re}(s)=\sigma} h\left(\frac{s}{i}\right)\left(\sum_{\chi \bmod q} \chi(l) \omega(s, \chi)\right) e^{-s x} d s \\
& -\sum_{\chi \bmod q} \chi(l) R(x, \chi) .
\end{aligned}
$$

It remains to estimate the two sums over $\chi$ on the right-hand side of (3.7). First we prove that in (3.7),

$$
\sum_{\chi \bmod q} \chi(l) \omega(s, \chi) \ll \log q .
$$

Note that this is much better than (3.4). 
We have remarked that in (3.3), the $M$ terms arise only for $\chi^{0} \bmod q$. Therefore,

$$
\begin{aligned}
\sum_{\chi \bmod q} \chi(l)\left\{\frac{M^{\prime}}{M}\right. & \left.\left(\frac{1}{2}+\sigma T+i t T, \chi\right)+\frac{M^{\prime}}{M}\left(\frac{1}{2}-\sigma T-i t T, \chi\right)\right\} \\
& =\frac{M^{\prime}}{M}\left(\frac{1}{2}+\sigma T+i t T, \chi^{0}\right)+\frac{M^{\prime}}{M}\left(\frac{1}{2}-\sigma T-i t T, \chi^{0}\right) \\
& =\frac{\log q}{q^{1 / 2+\sigma T+i t T}-1}+\frac{\log q}{q^{1 / 2-\sigma T-i t T}-1} \\
& \ll \log q .
\end{aligned}
$$

On the other hand,

$$
\begin{aligned}
\sum_{\chi \bmod q} \chi(l)\{ & \left.\frac{\Gamma_{\mathbb{R}}^{\prime}}{\Gamma_{\mathbb{R}}}\left(\frac{1}{2}+\mu(\chi)+s T\right)+\frac{\Gamma_{\mathbb{R}}^{\prime}}{\Gamma_{\mathbb{R}}}\left(\frac{1}{2}+\mu(\chi)-s T\right)\right\} \\
= & \left\{\sum_{\substack{\chi \bmod q \\
\chi(-1)=1}} \chi(l)\right\}\left\{\frac{\Gamma_{\mathbb{R}}^{\prime}}{\Gamma_{\mathbb{R}}}\left(\frac{1}{2}+s T\right)+\frac{\Gamma_{\mathbb{R}}^{\prime}}{\Gamma_{\mathbb{R}}}\left(\frac{1}{2}-s T\right)\right\} \\
& +\left\{\sum_{\substack{\chi \bmod q \\
\chi(-1)=-1}} \chi(l)\right\}\left\{\frac{\Gamma_{\mathbb{R}}^{\prime}}{\Gamma_{\mathbb{R}}}\left(\frac{3}{2}+s T\right)+\frac{\Gamma_{\mathbb{R}}^{\prime}}{\Gamma_{\mathbb{R}}}\left(\frac{3}{2}-s T\right)\right\}=0,
\end{aligned}
$$

by Lemma 3.2. Finally, we note that, under the condition of the lemma (i.e. $q$ is prime), all nonprincipal characters modulo $q$ are primitive. Thus,

$$
\begin{aligned}
\sum_{\chi \bmod q} \chi(l) \log \operatorname{cond}(\chi) & =\chi^{0}(l) \log 1+\sum_{\chi \neq \chi^{0} \bmod q} \chi(l) \log q \\
& =(\log q) \sum_{\chi \neq \chi^{0} \bmod q} \chi(l) \\
& =-\log q .
\end{aligned}
$$

Collecting all the estimates into the definition of $\omega(s, \chi)$ in (3.3), we get (3.8). Secondly we estimate the last sum over $\chi$ in (3.7). By (3.6), we have

$$
\begin{aligned}
\sum_{\chi \bmod q} \chi(l) R(x, \chi)= & \frac{1}{T} \sum_{0 \leq k \leq \sigma T / 2} \sum_{\chi \bmod q} \chi(l) h\left(\frac{s_{k}(\chi)}{i}\right) \exp \left(-s_{k}(\chi) x\right) \\
& +\sum_{m \in \mathbb{Z}} h\left(\frac{\varpi_{m}}{i}\right) \exp \left(-\varpi_{m} x\right) .
\end{aligned}
$$

Lemma 3.2 also gives 


$$
\begin{aligned}
\sum_{\chi \bmod q} \chi(l) h\left(\frac{s_{k}(\chi)}{i}\right) \exp \left(-s_{k}(\chi) x\right) \\
=\left\{\sum_{\substack{\chi \bmod q \\
\chi(-1)=1}} \chi(l)\right\} h\left(\frac{1 / 2+2 k}{T i}\right) \exp \left(-\frac{1 / 2+2 k}{T} x\right) \\
+\left\{\sum_{\substack{\chi \bmod q \\
\chi(-1)=-1}} \chi(l)\right\} h\left(\frac{3 / 2+2 k}{T i}\right) \exp \left(-\frac{3 / 2+2 k}{T} x\right)=0 .
\end{aligned}
$$

Clearly,

$$
\sum_{m \in \mathbb{Z}} h\left(\frac{\varpi_{m}}{i}\right) \exp \left(-\varpi_{m} x\right) \ll e^{-x /(2 T)},
$$

and therefore,

$$
\sum_{\chi \bmod q} \chi(l) R(x, \chi) \ll e^{-x /(2 T)} .
$$

Putting (3.8) and (3.9) into (3.7), we get

$$
\begin{aligned}
\sum_{\chi \bmod q} \chi(l) g_{T}(x, \chi) & \ll(\log q) \int_{\operatorname{Re}(s)=\sigma}\left|h\left(\frac{s}{i}\right)\right| e^{-\sigma x} d s+e^{-x /(2 T)} \\
& \ll e^{-\sigma x} \log q+e^{-x /(2 T)}
\end{aligned}
$$

and this is the required result.

4. Beginning of the proof of Theorem 1.1: Estimates for $C_{k_{1}, 0}$ and $C_{0, k_{2}}$. Let $h_{1}, g_{1}, h_{2}, g_{2}$ be as in Theorem 1.1. Applying Lemma 2.3, for $j=1,2$ we get

$$
\begin{aligned}
\sum_{\gamma_{j}} h_{j}\left(\frac{\gamma_{j}}{T}\right) e^{-i L \gamma u_{j}}= & T g_{j T}\left(T L u_{j}, \chi_{j}\right) \\
& +T Z_{j}^{+}\left(T, u_{j}, \chi_{j}\right)+T Z_{j}^{-}\left(T, u_{j}, \bar{\chi}_{j}\right) \\
& +\delta_{\chi_{j}} T J_{j}^{+}\left(T, u_{j}\right)+\delta_{\chi_{j}} T J_{j}^{-}\left(T, u_{j}\right) .
\end{aligned}
$$

Inserting the two formulae into the definition of $F\left(\mathbf{h}, T, \Phi ; \chi_{1}, \chi_{2}\right)$ in (1.3), we get 25 terms. In (4.1), if we enumerate the five terms on the right side respectively by $0,1,-1,2,-2$, then each of these 25 terms can be written as $C_{k_{1}, k_{2}}\left(\mathbf{h}, T, \Phi ; \chi_{1}, \chi_{2}\right)$ with $k_{1}, k_{2}=0, \pm 1, \pm 2$, which denotes the contribution from the product of the term $k_{1}$ of (4.1) for $j=1$ and term $k_{2}$ of (4.1) for $j=2$. For example,

$$
C_{1,-1}\left(\mathbf{h}, T, \Phi ; \chi_{1}, \chi_{2}\right)=\int_{\mathbb{R}}\left(T Z_{1}^{+}\left(T, u, \chi_{1}\right)\right)\left(T Z_{2}^{-}\left(T,-u, \bar{\chi}_{2}\right)\right) \Phi(u) d u .
$$


Thus, we get

$$
F\left(\mathbf{h}, T, \Phi ; \chi_{1}, \chi_{2}\right)=\sum_{\left|k_{1}\right|,\left|k_{2}\right| \leq 2} C_{k_{1}, k_{2}}\left(\mathbf{h}, T, \Phi ; \chi_{1}, \chi_{2}\right) .
$$

We will estimate $C_{k_{1}, k_{2}}\left(\mathbf{h}, T, \Phi ; \chi_{1}, \chi_{2}\right)$ separately in the following sections.

In this section we give estimates for $C_{k_{1}, 0}$ and $C_{0, k_{2}}$. We have

$$
C_{0,0}=T^{2} \int_{\mathbb{R}} g_{1 T}\left(T L u, \chi_{1}\right) g_{2 T}\left(-T L u, \chi_{2}\right) \Phi(u) d u .
$$

Actually the above integral is on $\operatorname{supp}(\Phi)=\left[b_{1}, b_{2}\right]$. By Lemma 3.1,

$$
\begin{aligned}
& \sum_{\chi_{1}, \chi_{2} \bmod q} \chi_{1}(l) \bar{\chi}_{2}(l) C_{0,0} \\
= & T^{2} \int_{b_{1}}^{b_{2}} \sum_{\chi_{1} \bmod q} \chi_{1}(l) g_{1 T}\left(T L u, \chi_{1}\right) \sum_{\chi_{2} \bmod q} \bar{\chi}_{2}(l) g_{2 T}\left(-T L u, \chi_{2}\right) \Phi(u) d u \\
\ll & T^{2} \int_{b_{1}}^{b_{2}}\left(e^{-2 T L u} \log ^{2} q+e^{-L u}\right) d u \ll \log ^{2} q+\frac{T^{2-b_{1}}}{L} \ll T^{2-b_{1}},
\end{aligned}
$$

where in the last step we have used $q \leq T^{b_{2}}$. However, by $T^{1-b_{1}} \leq q$, the above bound is

$$
T^{2-b_{1}} \ll q T \ll \varphi(q) T L .
$$

We will need the weighted Brun-Titchmarsh theorem in our future argument.

LEMMA 4.1. Let $x, y$ be any real numbers and $k, l$ integers satisfying $1 \leq k<y$ and $(k, l)=1$. Then

$$
\sum_{\substack{y<p \leq x \\ p \equiv l(\bmod k)}} \frac{\log p}{p} \ll \frac{\log ^{2} x}{\varphi(k) \log (y / k)} .
$$

Proof. By partial summation,

$$
\sum_{\substack{y<p \leq x \\ p \equiv l(\bmod k)}} \frac{\log p}{p}=\int_{y}^{x} \frac{\log t}{t} d \pi(t, k, l),
$$

where $\pi(t, k, l)$ denotes the number of primes $p \leq t$ with $p \equiv l(\bmod k)$. By the Brun-Titchmarsh theorem (see for example [3, Theorem 3.7]), if $1 \leq k<t,(k, l)=1$, then

$$
\pi(t, k, l)<\frac{3 t}{\varphi(k) \log (t / k)}
$$


From this and $k<y$, we deduce that

$$
\begin{aligned}
\sum_{\substack{y<p \leq x \\
p \equiv l(\bmod k)}} \frac{\log p}{p} & \ll \frac{\log x}{\varphi(k) \log (x / k)}+\frac{\log y}{\varphi(k) \log (y / k)} \\
& +\int_{y}^{x} \frac{\log t}{\varphi(k) t \log (t / k)} d t \\
& \ll \frac{\log ^{2} x}{\varphi(k) \log (y / k)} .
\end{aligned}
$$

This proves the lemma.

For $C_{0,1}$ we make the substitution $x=T(L u-\log n)$, to get

$$
\begin{aligned}
C_{0,1}= & -T^{2} \int_{\mathbb{R}} g_{1 T}\left(T L u, \chi_{1}\right) \sum_{n} w\left(n, \chi_{2}\right) g_{2}(T(-L u+\log n)) \Phi(u) d u \\
= & -\frac{T}{L} \int_{-a}^{a} g_{2}(-x) \sum_{n} w\left(n, \chi_{2}\right) \\
& \times g_{1 T}\left(x+T \log n, \chi_{1}\right) \Phi\left(\frac{x}{T L}+\frac{\log n}{L}\right) d x
\end{aligned}
$$

since $\operatorname{supp}\left(g_{j}\right)=[-a, a]$. Therefore,

$$
\begin{aligned}
& \sum_{\chi_{1}, \chi_{2} \bmod q} \chi_{1}(l) \bar{\chi}_{2}(l) C_{0,1} \\
& =-\frac{T}{L} \int_{-a}^{a} g_{2}(-x) \sum_{n} \Phi\left(\frac{x}{T L}+\frac{\log n}{L}\right) \sum_{\chi_{2} \bmod q} \bar{\chi}_{2}(l) w\left(n, \chi_{2}\right) \\
& \quad \times \sum_{\chi_{1} \bmod q} \chi_{1}(l) g_{1 T}\left(x+T \log n, \chi_{1}\right) d x
\end{aligned}
$$

Now we should have

$$
\frac{x}{T L}+\frac{\log n}{L} \in\left[b_{1}, b_{2}\right]=\operatorname{supp}(\Phi),
$$

but, for large $T$, this implies

$$
T^{b_{1}} / 2<n \leq 2 T^{b_{2}}
$$

since $x \in[-a, a]$. By Lemma 3.1, the sum over $\chi_{1}$ in (4.5) can be estimated as

$$
\begin{aligned}
\sum_{\chi_{1} \bmod q} \chi_{1}(l) g_{1 T}\left(x+T \log n, \chi_{1}\right) & \ll e^{-|x+T \log n| /(2 T)} \\
& \ll e^{(-T \log n+a) /(2 T)} \ll n^{-1 / 2} .
\end{aligned}
$$


For the sum over $\chi_{2}$ in (4.5), we use the definition of $w(n, \chi)$ in $(2.15)$ :

$$
\begin{aligned}
\sum_{\chi_{2} \bmod q} \bar{\chi}_{2}(l) w\left(n, \chi_{2}\right) & \\
& =\sum_{\chi_{2} \bmod q} \bar{\chi}_{2}(l)\left(\frac{\Lambda(n) \chi_{2}(n)}{\sqrt{n}}-\delta_{\chi_{2}}\left(\frac{1}{\sqrt{n}}+\frac{1}{n^{3 / 2}}\right)\right) \\
& = \begin{cases}\varphi(q) \frac{\Lambda(n)}{\sqrt{n}}-\left(\frac{1}{\sqrt{n}}+\frac{1}{n^{3 / 2}}\right) & \text { if } n \equiv l(\bmod q), \\
-\left(\frac{1}{\sqrt{n}}+\frac{1}{n^{3 / 2}}\right) & \text { otherwise. }\end{cases}
\end{aligned}
$$

Putting this and (4.7) into (4.5), we get

$$
\begin{aligned}
& \sum_{\chi_{1}, \chi_{2} \bmod q} \chi_{1}(l) \bar{\chi}_{2}(l) C_{0,1} \\
& \ll \frac{T}{L} \int_{-a}^{a}\left|g_{2}(-x)\right| d x \sum_{n}\left|\sum_{\chi_{2} \bmod q} \bar{\chi}_{2}(l) w\left(n, \chi_{2}\right)\right| \\
& \times\left|\sum_{\chi_{1} \bmod q} \chi_{1}(l) g_{1 T}\left(x+T \log n, \chi_{1}\right)\right| \\
& \ll \varphi(q) \frac{T}{L} \sum_{\substack{T^{b_{1}} / 2<n \leq 2 T^{b_{2}} \\
n \equiv l(\bmod q)}} \frac{\Lambda(n)}{n}+\frac{T}{L} \sum_{T^{b_{1} / 2<n \leq 2 T^{b_{2}}}} \frac{1}{n} \ll T
\end{aligned}
$$

by Lemma 4.1 and the assumption $q \leq T^{b_{1}-\varepsilon}$.

The corresponding estimates for $C_{0,-1}, C_{ \pm 1,0}$ follow the same lines, and their upper bounds are just the same as in (4.9).

For $C_{0,2}$, we make the substitution $x=T(L u-\log v)$, to get

$$
\begin{aligned}
\sum_{\chi_{1} \bmod q} \chi_{1}(l) C_{0,2}= & \delta_{\chi_{2}} T^{2} \int_{\mathbb{R}} \sum_{\chi_{1} \bmod q} \chi_{1}(l) g_{1 T}\left(T L u, \chi_{1}\right)\left\{\int_{1}^{\infty}\left(\frac{1}{\sqrt{v}}+\frac{1}{v^{3 / 2}}\right)\right. \\
& \left.\times g_{2}(T(-L u+\log v)) d(v-[v])\right\} \Phi(u) d u \\
= & \delta_{\chi_{2}} \frac{T}{L} \int_{\mathbb{R}} g_{2}(-x)\left\{\int_{1}^{\infty}\left(\frac{1}{\sqrt{v}}+\frac{1}{v^{3 / 2}}\right) \Phi\left(\frac{x}{T L}+\frac{\log v}{L}\right)\right. \\
& \left.\times \sum_{\chi_{1} \bmod q} \chi_{1}(l) g_{1 T}\left(x+T \log v, \chi_{1}\right) d(v-[v])\right\} d x .
\end{aligned}
$$

By (4.6) and (4.7), this is 
and therefore,

$$
\ll \delta_{\chi_{2}} \frac{T}{L} \int_{-a}^{a}\left|g_{2}(-x)\right| \int_{T^{b_{1} / 2}}^{2 T^{b_{2}}} \frac{1}{v} d(v-[v]) d x \ll \delta_{\chi_{2}} \frac{T}{L},
$$

$$
\sum_{\chi_{1}, \chi_{2} \bmod q} \chi_{1}(l) \bar{\chi}_{2}(l) C_{0,2} \ll \frac{T}{L} .
$$

The same bound holds also for the corresponding sums for $C_{0,-2}$ and $C_{ \pm 2,0}$.

Summarizing the estimates (4.3), (4.4), (4.9), and (4.10), we get

$$
\sum_{\chi_{1} \bmod q} \chi_{1}(l) \bar{\chi}_{2}(l) \sum_{k_{1} \text { or } k_{2}=0} C_{k_{1}, k_{2}} \ll \varphi(q) T L .
$$

5. Estimates for $C_{ \pm 1, \pm 1}$. We begin with $C_{1,1}$. Recall that

$$
\begin{aligned}
C_{1,1}= & T^{2} \int_{\mathbb{R}} \sum_{n_{1}, n_{2}} w\left(n_{1}, \chi_{1}\right) w\left(n_{2}, \chi_{2}\right) \\
& \times g_{1}\left(T\left(L u+\log n_{1}\right)\right) g_{2}\left(T\left(-L u+\log n_{2}\right)\right) \Phi(u) d u \\
= & \frac{T}{L} \int_{-a}^{a} g_{1}(x) \sum_{n_{1}, n_{2}} w\left(n_{1}, \chi_{1}\right) w\left(n_{2}, \chi_{2}\right) \\
& \times g_{2}\left(-x+T \log \left(n_{1} n_{2}\right)\right) \Phi\left(\frac{x}{T L}-\frac{\log n_{1}}{L}\right) d x,
\end{aligned}
$$

where $x=T\left(L u+\log n_{1}\right)$ is our new variable. We need to have $-a \leq x \leq a$ and $-a \leq-x+T \log \left(n_{1} n_{2}\right) \leq a$ in order to have a nonzero integral, but when $T$ is sufficiently large this is impossible unless $n_{1}=n_{2}=1$. Thus,

$$
C_{1,1}=4 \delta_{\chi_{1}} \delta_{\chi_{2}} \frac{T}{L} \int_{-a}^{a} g_{1}(x) g_{2}(-x) \Phi\left(\frac{x}{T L}\right) d x .
$$

Since $x /(T L)<b_{1}$ for large $T$, we have

$$
C_{1,1}=0 \text {. }
$$

The same result is true for $C_{-1,-1}$.

Letting $x=T\left(L u-\log n_{1}\right)$, we have

$$
\begin{aligned}
C_{-1,1}= & T^{2} \int_{\mathbb{R}} \sum_{n_{1}, n_{2}} w\left(n_{1}, \bar{\chi}_{1}\right) w\left(n_{2}, \chi_{2}\right) \\
& \times g_{1}\left(T\left(L u-\log n_{1}\right)\right) g_{2}\left(T\left(-L u+\log n_{2}\right)\right) \Phi(u) d u \\
= & \frac{T}{L} \int_{-a}^{a} g_{1}(x) \sum_{n_{1}, n_{2}} w\left(n_{1}, \bar{\chi}_{1}\right) w\left(n_{2}, \chi_{2}\right) \\
& \times g_{2}\left(-x+T \log \frac{n_{2}}{n_{1}}\right) \Phi\left(\frac{x}{T L}+\frac{\log n_{1}}{L}\right) d x .
\end{aligned}
$$


Thus, by (4.6),

$$
\begin{aligned}
\sum_{\chi_{1}, \chi_{2} \bmod q} \chi_{1}(l) & \bar{\chi}_{2}(l) C_{-1,1} \\
= & \frac{T}{L} \int_{-a}^{a} g_{1}(x) \sum_{n_{1}} \sum_{\chi_{1} \bmod q} \chi_{1}(l) w\left(n_{1}, \bar{\chi}_{1}\right) \Phi\left(\frac{x}{T L}+\frac{\log n_{1}}{L}\right) \\
& \times \sum_{n_{2}} \sum_{\chi_{2} \bmod q} \bar{\chi}_{2}(l) w\left(n_{2}, \chi_{2}\right) g_{2}\left(-x+T \log \frac{n_{2}}{n_{1}}\right) d x
\end{aligned}
$$

which is

$$
\begin{aligned}
& \ll \frac{T}{L} \int_{-a}^{a}\left|g_{1}(x)\right| \sum_{T^{b_{1}} / 2<n_{1} \leq 2 T^{b_{2}}}\left|\sum_{\chi_{1} \bmod q} \chi_{1}(l) w\left(n_{1}, \bar{\chi}_{1}\right)\right| \\
& \quad \times\left|\sum_{n_{2}} \sum_{\chi_{2} \bmod q} \bar{\chi}_{2}(l) w\left(n_{2}, \chi_{2}\right) g_{2}\left(-x+T \log \frac{n_{2}}{n_{1}}\right)\right| d x .
\end{aligned}
$$

As before, we should have $-a \leq x \leq a$ and $-a \leq-x+T \log \left(n_{2} / n_{1}\right) \leq a$, which implies that

$$
n_{1} e^{-2 a / T} \leq n_{2} \leq n_{1} e^{2 a / T}
$$

and hence by $b_{2}<1$,

$$
\left|n_{2}-n_{1}\right| \ll \frac{n_{1}}{T} \ll T^{b_{2}-1} \rightarrow 0 \quad \text { as } T \rightarrow \infty .
$$

Therefore we must have $n_{2}=n_{1}$, and consequently by (2.15) and (4.8),

$$
\begin{aligned}
& \sum_{\chi_{1}, \chi_{2} \bmod q} \chi_{1}(l) \bar{\chi}_{2}(l) C_{-1,1} \\
& \ll \frac{T}{L} \sum_{T^{b_{1}} / 2<n \leq 2 T^{b_{2}}}\left|\sum_{\chi_{1} \bmod q} \chi_{1}(l) w\left(n, \bar{\chi}_{1}\right)\right|\left|\sum_{\chi_{2} \bmod q} \bar{\chi}_{2}(l) w\left(n, \chi_{2}\right)\right| \\
& \times \int_{-a}^{a}\left|g_{1}(x) g_{2}(-x)\right| d x
\end{aligned}
$$

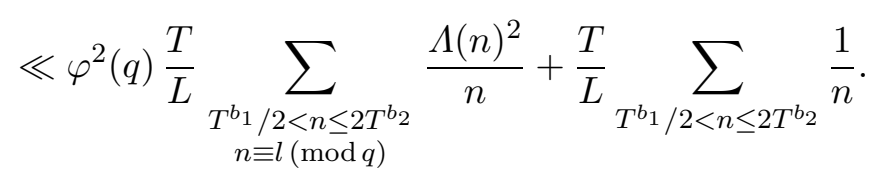

We use again Lemma 4.1 and the assumption $q \leq T^{b_{1}-\varepsilon}$, to get

$$
\sum_{\chi_{1}, \chi_{2} \bmod q} \chi_{1}(l) \bar{\chi}_{2}(l) C_{-1,1} \ll \varphi(q) T L .
$$

The upper bound for the corresponding mean value for $C_{1,-1}$ is the same. 
We conclude from (5.1) and (5.2) that

$$
\sum_{\chi_{1}, \chi_{2} \bmod q} \chi_{1}(l) \bar{\chi}_{2}(l) \sum_{\substack{k_{1}= \pm 1 \\ k_{2}= \pm 1}} C_{k_{1}, k_{2}} \ll \varphi(q) T L .
$$

6. Estimates for the other $C_{k_{1}, k_{2}}$. We have

$$
\begin{aligned}
C_{1,2}= & -\delta_{\chi_{2}} T^{2} \int_{\mathbb{R}} \sum_{n} w\left(n, \chi_{1}\right) g_{1}(T(L u+\log n)) \\
& \times \int_{1}^{\infty} g_{2}(T(-L u+\log v))\left(\frac{1}{\sqrt{v}}+\frac{1}{v^{3 / 2}}\right) d(v-[v]) \Phi(u) d u \\
= & -\delta_{\chi_{2}} \frac{T}{L} \int_{-a}^{a} g_{1}(x) \sum_{n} w\left(n, \chi_{1}\right) \int_{1}^{\infty} g_{2}(-x+T \log (n v)) \\
& \times\left(\frac{1}{\sqrt{v}}+\frac{1}{v^{3 / 2}}\right) d(v-[v]) \Phi\left(\frac{x}{T L}-\frac{\log n}{L}\right) d u,
\end{aligned}
$$

by changing variables $x=T(L u+\log n)$. For large $T$, the inequalities $-a \leq x \leq a$ and $-a \leq-x+T \log (n v) \leq a$ are impossible unless $n=1$. Consequently,

$$
\begin{aligned}
C_{1,2}= & -2 \delta_{\chi_{2}} \frac{T}{L} \int_{-a}^{a} g_{1}(x) \Phi\left(\frac{x}{T L}\right) \\
& \times \int_{1}^{\infty} g_{2}(-x+T \log v)\left(\frac{1}{\sqrt{v}}+\frac{1}{v^{3 / 2}}\right) d(v-[v]) d x .
\end{aligned}
$$

Since $x$ is bounded and $T$ large, we have $x /(T L)<b_{1}$, and therefore,

$$
C_{1,2}=0 \text {. }
$$

The same result is true for $C_{2,1}, C_{-1,-2}$, and $C_{-2,-1}$.

To estimate $C_{-1,2}$, we note that

$$
\begin{aligned}
C_{-1,2}= & -\delta_{\chi_{2}} T^{2} \int_{\mathbb{R}} \sum_{n} w\left(n, \bar{\chi}_{1}\right) g_{1}(T(L u-\log n)) \\
& \times \int_{1}^{\infty} g_{2}(T(-L u+\log v))\left(\frac{1}{\sqrt{v}}+\frac{1}{v^{3 / 2}}\right) \Phi(u) d(v-[v]) d u \\
= & -\delta_{\chi_{2}} \frac{T}{L} \int_{\mathbb{R}} g_{2}(x) \sum_{n} w\left(n, \bar{\chi}_{1}\right) \int_{1}^{\infty} g_{1}\left(-x+T \log \frac{v}{n}\right) \\
& \times\left(\frac{1}{\sqrt{v}}+\frac{1}{v^{3 / 2}}\right) d(v-[v]) \Phi\left(-\frac{x}{T L}+\frac{\log n}{L}\right) d x,
\end{aligned}
$$


which is

$$
\ll \delta_{\chi_{2}} \frac{T}{L} \int_{-a}^{a} \int_{T^{b_{1} / 2}}^{2 T^{b_{2}}} \sum_{n}\left|w\left(n, \bar{\chi}_{1}\right)\right|\left|g_{1}\left(-x+T \log \frac{v}{n}\right)\right| \frac{1}{\sqrt{v}} d(v-[v]) d x .
$$

Note that the inequalities $-a \leq x \leq a$ and $-a \leq x+\log (v / n) \leq a$ imply

$$
v e^{-2 a / T} \leq n \leq v e^{2 a / T} \text {. }
$$

There is at most one $n$ in the above interval, since the length of the interval is

$$
v e^{2 a / T}-v e^{-2 a / T} \ll \frac{v}{T} \ll T^{b_{2}-1} \rightarrow 0 \quad \text { as } T \rightarrow \infty .
$$

Therefore,

$$
\sum_{v e^{-2 a / T} \leq n \leq v e^{2 a / T}}\left|w\left(n, \bar{\chi}_{1}\right)\right|\left|g_{1}\left(x+T \log \frac{v}{n}\right)\right| \ll \sum_{v e^{-2 a / T} \leq n \leq v e^{2 a / T}} \frac{\log n}{\sqrt{n}} \ll \frac{\log v}{\sqrt{v}},
$$

and consequently,

$$
C_{-1,2} \ll \delta_{\chi_{2}} \frac{T}{L} \int_{-a}^{a} \int_{T^{b_{1} / 2}}^{2 T^{b_{2}}} \frac{\log v}{v} d(v-[v]) d x \ll \delta_{\chi_{2}} T^{1-b_{1}} .
$$

Hence

$$
\sum_{\chi_{1}, \chi_{2} \bmod q} \chi_{1}(l) \bar{\chi}_{2}(l) C_{-1,2} \ll \varphi(q) T^{1-b_{1}} .
$$

The corresponding sums for $C_{1,-2}, C_{2,-1}$, and $C_{-2,1}$ have the same upper bound estimate.

Now we turn to $C_{2,2}$ :

$$
\begin{aligned}
C_{2,2}= & \delta_{\chi_{1}} \delta_{\chi_{2}} T^{2} \int_{\mathbb{R}} \Phi(u) d u \\
& \times \int_{1}^{\infty} \int_{1}^{\infty} g_{1}\left(T\left(L u+\log v_{1}\right)\right) g_{2}\left(T\left(-L u+\log v_{2}\right)\right) \\
& \times\left(\frac{1}{\sqrt{v_{1}}}+\frac{1}{v_{1}^{3 / 2}}\right)\left(\frac{1}{\sqrt{v_{2}}}+\frac{1}{v_{2}^{3 / 2}}\right) d\left(v_{1}-\left[v_{1}\right]\right) d\left(v_{2}-\left[v_{2}\right]\right) .
\end{aligned}
$$

On changing variables this is seen to be

$$
\begin{aligned}
= & \delta_{\chi_{1}} \delta_{\chi_{2}} \frac{T}{L} \int_{\mathbb{R}} g_{1}(x) d x \\
& \times \int_{1}^{\infty} \int_{1}^{\infty} g_{2}\left(-x+T \log \left(v_{1} v_{2}\right)\right) \Phi\left(\frac{x}{T L}-\frac{\log v_{1}}{L}\right) \\
& \times\left(\frac{1}{\sqrt{v_{1}}}+\frac{1}{v_{1}^{3 / 2}}\right)\left(\frac{1}{\sqrt{v_{2}}}+\frac{1}{v_{2}^{3 / 2}}\right) d\left(v_{1}-\left[v_{1}\right]\right) d\left(v_{2}-\left[v_{2}\right]\right) .
\end{aligned}
$$


We should have $T^{b_{1}} / 2 \leq v_{1} \leq 2 T^{b_{2}}$ because of the support of $\Phi$. Therefore, the two inequalities $-a \leq x \leq a$ and $-a \leq-x+T \log \left(v_{1} v_{2}\right) \leq a$ cannot hold at the same time. Thus,

$$
C_{2,2}=0 \text {. }
$$

Similarly $C_{-2,-2}=0$.

Using the method for $C_{-1,1}$ we have

$$
\begin{aligned}
C_{-2,2}= & \delta_{\chi_{1}} \delta_{\chi_{2}} T^{2} \int_{\mathbb{R}} \Phi(u) d u \\
& \times \int_{1}^{\infty} \int_{1}^{\infty} g_{1}\left(T\left(L u-\log v_{1}\right)\right) g_{2}\left(T\left(-L u+\log v_{2}\right)\right) \\
& \times\left(\frac{1}{\sqrt{v_{1}}}+\frac{1}{v_{1}^{3 / 2}}\right)\left(\frac{1}{\sqrt{v_{2}}}+\frac{1}{v_{2}^{3 / 2}}\right) d\left(v_{1}-\left[v_{1}\right]\right) d\left(v_{2}-\left[v_{2}\right]\right) \\
= & \delta_{\chi_{1}} \delta_{\chi_{2}} \frac{T}{L} \int_{\mathbb{R}} g_{1}(x) d x \\
& \times \int_{1}^{\infty} \int_{1}^{\infty} g_{2}\left(-x+T \log \frac{v_{2}}{v_{1}}\right) \Phi\left(\frac{x}{T L}+\frac{\log v_{1}}{L}\right) \\
& \times\left(\frac{1}{\sqrt{v_{1}}}+\frac{1}{v_{1}^{3 / 2}}\right)\left(\frac{1}{\sqrt{v_{2}}}+\frac{1}{v_{2}^{3 / 2}}\right) d\left(v_{1}-\left[v_{1}\right]\right) d\left(v_{2}-\left[v_{2}\right]\right) \\
\ll & \delta_{\chi_{1}} \delta_{\chi_{2}} \frac{T}{L} \int_{T^{b_{1}} / 2}^{2 T^{b_{2}}} \int_{1}^{\infty} \frac{d\left(v_{1}-\left[v_{1}\right]\right) d\left(v_{2}-\left[v_{2}\right]\right)}{v_{1}^{1 / 2} v_{2}^{1 / 2}} \\
& \times \int_{-a}^{a}\left|g_{1}(x) g_{2}\left(-x+T \log \frac{v_{2}}{v_{1}}\right)\right| d x .
\end{aligned}
$$

Appealing to (6.2), we get

$$
\begin{aligned}
C_{-2,2} & \ll \delta_{\chi_{1}} \delta_{\chi_{2}} \frac{T}{L} \int_{T^{b_{1} / 2}}^{2 T^{b_{2}}} \frac{d\left(v_{1}-\left[v_{1}\right]\right)}{v_{1}^{1 / 2}} \int_{v_{1} e^{-2 a / T}}^{v_{1} e^{2 a / T}} \frac{d\left(v_{2}-\left[v_{2}\right]\right)}{v_{2}^{1 / 2}} \\
& \ll \delta_{\chi_{1}} \delta_{\chi_{2}} \frac{T}{L} \int_{T^{b_{1} / 2}}^{2 T^{b_{2}}} \frac{d\left(v_{1}-\left[v_{1}\right]\right)}{v_{1}} \ll \delta_{\chi_{1}} \delta_{\chi_{2}} \frac{T}{L} .
\end{aligned}
$$

Therefore,

$$
\sum_{\chi_{1}, \chi_{2} \bmod q} \chi_{1}(l) \bar{\chi}_{2}(l) C_{-2,2} \ll \frac{T}{L} .
$$

The same upper bound holds for the corresponding sum for $C_{2,-2}$. 
Summarizing the estimates (6.1), and (6.3)-(6.5), we get

$$
\sum_{\chi_{1}, \chi_{2} \bmod q} \chi_{1}(l) \bar{\chi}_{2}(l) \sum_{k_{1}, k_{2}= \pm 1 \text { or } \pm 2} C_{k_{1}, k_{2}} \ll \frac{T}{L} .
$$

Now we can complete the proof of Theorem 1.1 by inserting the estimates from (4.11), (5.3), and (6.6) into (4.2).

7. Distribution of primes in arithmetic progressions. Now we study the distribution of primes in arithmetic progressions. Let $\xi>1$, whose value will be specified later. Let $g(x)$ be a function satisfying

$$
\operatorname{supp}(g)=[-1,1], \quad g(x)>0 \quad \text { for } x \in(-1,1)
$$

in addition to (1.1), and let $h(r)$ be as in (1.2). Now we define

$$
\Psi(T, \xi ; q, l)=T \sum_{\substack{n=1 \\ n \equiv l(\bmod q)}}^{\infty} \frac{\Lambda(n)}{\sqrt{n}} g(T(L \xi-\log n)) .
$$

In (7.2), the $n$ has to satisfy $-1 \leq T L \xi-T \log n \leq 1$, that is,

$$
T^{\xi} e^{-1 / T} \leq n \leq T^{\xi} e^{1 / T} .
$$

The length of the interval (7.3) is

$$
T^{\xi}\left(e^{1 / T}-e^{-1 / T}\right) \sim 2 T^{\xi-1},
$$

and therefore $\Psi(T, \xi ; q, l)$ is a weighted function counting primes in the arithmetic progression $n \equiv l(\bmod q)$ and in the interval (7.3) with the short length (7.4).

Introducing the Dirichlet characters, we have

$$
\Psi(T, \xi ; q, l)=-\frac{1}{\varphi(q)} \sum_{\chi \bmod q} \chi(l) T S^{-}(T, \xi, \bar{\chi}),
$$

where $S^{-}(T, \xi, \bar{\chi})$ is defined as in $(2.9)$.

Let $1 / 2+i \gamma$ go over the zeros of $L(s, \chi)$ within $0<\operatorname{Re}(s)<1$. Now we apply the explicit formula (2.7). For large $T$, the inequality $-1 \leq T L \xi+$ $\log n \leq 1$ is impossible, hence $T S^{+}(T, \xi, \chi)=0$, and consequently,

$$
-T S^{-}(T, \xi, \bar{\chi})=\delta_{\chi} P(T, \xi)-\sum_{\gamma} h\left(\frac{\gamma}{T}\right) e^{-i L \gamma \xi}+T g_{T}(T L \xi, \chi) .
$$

By Lemma 3.1,

$$
\sum_{\chi \bmod q} \chi(l) g_{T}(T L \xi, \chi) \ll e^{-T L \xi} \log q+e^{-L \xi / 2} \ll T^{-\xi / 2},
$$


if we assume $\log q \leq T^{\delta}$ for some positive $\delta$. This together with (7.5) gives

$$
\begin{aligned}
\Psi(T, \xi ; q, l)= & \frac{1}{\varphi(q)} P(T, \xi)-\frac{1}{\varphi(q)} \sum_{\chi \bmod q} \chi(l) \sum_{\gamma} h\left(\frac{\gamma}{T}\right) e^{-i L \gamma \xi} \\
& +O\left(\frac{T^{1-\xi / 2}}{\varphi(q)}\right) .
\end{aligned}
$$

Let

$$
\Sigma(v)=\Sigma(v ; h, T, \xi)=\sum_{\chi \bmod q} \chi(l) \sum_{\gamma} h\left(\frac{\gamma}{T}\right) e^{-i \gamma L(\xi-v)} .
$$

Obviously $\Sigma(0)$ is the sum over $\gamma$ in (7.6); it will be bounded via GRH and Conjecture 1.2.

Lemma 7.1. Let $h, g$ satisfy (1.1), (1.2), and (7.1). Then for $q<T^{\xi-1-\varepsilon}$,

$$
\Sigma(0) \ll \varphi(q)^{1 / 2} T L^{1 / 2} .
$$

Proof. The Sobolev-Gallagher inequality states that if $f(u)$ has a continuous derivative on $[-1 / 2,1 / 2]$, then

$$
|f(0)| \ll \int_{-1 / 2}^{1 / 2}\left|f^{\prime}(u)\right| d u+\int_{-1 / 2}^{1 / 2}|f(u)| d u .
$$

Suppose $\phi \in C_{\mathrm{c}}^{1}(\mathbb{R})$ with $\operatorname{supp}(\phi)=[-1,1]$. Then

$$
\begin{aligned}
\Sigma(0)^{2} \ll & \int_{-1 / 2}^{1 / 2}|\Sigma(v)|\left|\Sigma^{\prime}(v)\right| d v+\int_{-1 / 2}^{1 / 2}|\Sigma(v)|^{2} d v \\
\ll & \left(\int_{\mathbb{R}}|\Sigma(v)|^{2} \phi(v) d v\right)^{1 / 2}\left(\int_{\mathbb{R}}\left|\Sigma^{\prime}(v)\right|^{2} \phi(v) d v\right)^{1 / 2} \\
& +\int_{\mathbb{R}}|\Sigma(v)|^{2} \phi(v) d v .
\end{aligned}
$$

We need to estimate the integrals on the right hand side of (7.7). First,

$$
\begin{aligned}
\int_{\mathbb{R}}|\Sigma(v)|^{2} \phi(v) d v= & \sum_{\chi_{1}, \chi_{2} \bmod q} \chi_{1} \bar{\chi}_{2}(l) \sum_{\gamma_{1}, \gamma_{2}} h\left(\frac{\gamma_{1}}{T}\right) \bar{h}\left(\frac{\gamma_{2}}{T}\right) \\
& \times \int_{\mathbb{R}} e^{-i\left(\gamma_{1}-\gamma_{2}\right) L(\xi-v)} \phi(v) d v \\
= & \sum_{\chi_{1}, \chi_{2} \bmod q} \chi_{1} \bar{\chi}_{2}(l) \sum_{\gamma_{1}, \gamma_{2}} h\left(\frac{\gamma_{1}}{T}\right) \bar{h}\left(\frac{\gamma_{2}}{T}\right) \\
& \times \int_{\mathbb{R}} e^{-i\left(\gamma_{1}-\gamma_{2}\right) L w} \phi(\xi+w) d w .
\end{aligned}
$$


Letting $\Phi(v)=\phi(\xi+v)$, we get $\Phi \in C_{\mathrm{c}}^{1}(\mathbb{R})$ with $\operatorname{supp}(\Phi)=[\xi-1, \xi+1]$, and therefore the last integral above equals $f\left(L \gamma_{1} /(2 \pi), L \gamma_{2} /(2 \pi)\right)$ by $\S 1$. By GRH, all the $\gamma$ above are real, and so by $(1.2)$ we have $\bar{h}(r)=h(-r)$. Let $\mathbf{h}(r)=(h(r), h(-r))$. Then by (1.3),

$$
\int_{\mathbb{R}}|\Sigma(v)|^{2} \phi(v) d v=\sum_{\chi_{1}, \chi_{2} \bmod q} \chi_{1} \bar{\chi}_{2}(l) F\left(\mathbf{h}, T, \Phi ; \chi_{1}, \chi_{2}\right) .
$$

Since $q \leq T^{\xi-1-\varepsilon}$, we can apply Conjecture 1.2 to (7.8), to get

$$
\int_{\mathbb{R}}|\Sigma(v)|^{2} \phi(v) d v \ll \varphi(q) T L .
$$

To estimate the other integral on the right-hand side of (7.7), we note that

$$
\begin{aligned}
& \int_{\mathbb{R}}\left|\Sigma^{\prime}(v)\right|^{2} \phi(v) d v \\
= & \int_{\mathbb{R}}\left|\sum_{\chi \bmod q} \chi(l) \sum_{\gamma} h\left(\frac{\gamma}{T}\right) \gamma e^{-i \gamma L(\xi-v)}\right|^{2} \phi(v) d v \\
= & \sum_{\chi_{1}, \chi_{2} \bmod q} \chi_{1} \bar{\chi}_{2}(l) \sum_{\gamma_{1}, \gamma_{2}} h\left(\frac{\gamma_{1}}{T}\right) \bar{h}\left(\frac{\gamma_{2}}{T}\right) \gamma_{1} \gamma_{2} f\left(\frac{L}{2 \pi} \gamma_{1}, \frac{L}{2 \pi} \gamma_{2}\right) .
\end{aligned}
$$

By (1.1) and (1.2),

$$
g(x)=\int_{\mathbb{R}} h(r) e^{i x r} d r
$$

and consequently

$$
g^{\prime}(x)=\int_{\mathbb{R}} i h(r) r e^{i x r} d r
$$

Let $H(r)=i h(r) r$. Then

$$
H(r)=\int_{\mathbb{R}} g^{\prime}(x) e^{-i r x} d x
$$

and so $i \bar{h}(r) r=i h(-r) r=-H(-r)$, where we have used GRH and $\bar{h}(r)=$ $h(-r)$ as before. Now let $\mathbf{H}=(H(r),-H(-r))$. Then the right-hand side of (7.10) becomes

$$
-T^{2} \sum_{\chi_{1}, \chi_{2} \bmod q} \chi_{1} \bar{\chi}_{2}(l) F\left(\mathbf{H}, T, \Phi ; \chi_{1}, \chi_{2}\right),
$$

to which Conjecture 1.2 also applies. Thus, by an argument similar to that leading to (7.9),

$$
\int_{\mathbb{R}}\left|\Sigma^{\prime}(v)\right|^{2} \phi(v) d v \ll \varphi(q) T^{3} L
$$


Inserting this and (7.9) into (7.7), we get $\Sigma(0)^{2} \ll \varphi(q) T^{2} L$. This proves the lemma.

From (7.6) and Lemma 7.1, we conclude the following weighted prime number theorem in arithmetic progressions in short intervals.

TheOREm 7.2. Assume GRH and Conjecture 1.2. Let $q$ be prime and $(l, q)=1$ with $l \not \equiv \pm 1(\bmod q)$. Let $h$ and $g$ satisfy (1.1), (1.2), and (7.1). Then, uniformly for $\xi>1$,

$$
\Psi(T, \xi ; q, l)=\frac{P(T, \xi)}{\varphi(q)}+O\left(\frac{T L^{1 / 2}}{\varphi(q)^{1 / 2}}+\frac{T^{1-\xi / 2}}{\varphi(q)}\right) .
$$

Theorem 1.3 is a corollary of Theorem 7.2.

Proof of Theorem 1.3. The main term in Theorem 7.2 is

$$
\frac{P(T, \xi)}{\varphi(q)} \sim h(0) \frac{T^{\xi / 2}}{\varphi(q)},
$$

where

$$
h(0)=\int_{\mathbb{R}} g(x) d x>0
$$

by (1.2) and (7.1). Let $\xi>2$ be arbitrary, and $q$ large. Then one can always find $T$ such that

$$
\begin{aligned}
2 C\left(\frac{T L^{1 / 2}}{\varphi^{1 / 2}(q)}+\frac{T^{1-\xi / 2}}{\varphi(q)}\right) & \leq h(0) \frac{T^{\xi / 2}}{\varphi(q)} \\
& \leq 3 C\left(\frac{T L^{1 / 2}}{\varphi^{1 / 2}(q)}+\frac{T^{1-\xi / 2}}{\varphi(q)}\right),
\end{aligned}
$$

where $C$ is the constant implied in the error term in Theorem 7.2. For this $T$, Theorem 7.2 and the first inequality in $(7.12)$ assert that $\Psi(T, \xi ; q, l)>0$, that is, there is a prime $p_{0} \equiv l(\bmod q)$ lying in the interval $(7.3)$. From this and the second inequality in (7.12), we conclude that

$$
p_{0} \leq T^{\xi} e^{1 / T} \ll \varphi(q)^{\xi /(\xi-2)} \log q .
$$

The desired result now follows from choosing a suitable $\xi$.

\section{References}

[1] H. Davenport, Multiplicative Number Theory, Markham, Chicago, 1967.

[2] A. Granville and C. Pomerance, On the least prime in certain arithmetic progressions, J. London Math. Soc. (2) 41 (1990), 193-200.

[3] H. Halberstam and H.-E. Richert, Sieve Methods, Academic Press, London, 1974.

[4] D. R. Heath-Brown, Zero-free regions for Dirichlet L-functions, and the least prime in an arithmetic progression, Proc. London Math. Soc. (3) 64 (1992), 265-338. 
[5] C. P. Hughes and Z. Rudnick, Linear statistics of low-lying zeros of L-functions, Quart. J. Math. 54 (2003), 309-333.

[6] A. Languasco and A. Perelli, A pair correlation hypothesis and the exceptional set in Goldbach's problem, Mathematika 43 (1996), 349-361.

[7] Yu. V. Linnik, On the least prime in an arithmetic progression, I: the basic theorem, Math. Sb. (N.S.) 15 (1944), 139-178.

[8] - On the least prime in an arithmetic progression, II: the Deuring-Heilbronn phenomenon, ibid., 347-368.

[9] J. Y. Liu and Y. Ye, Superposition of zeros of distinct L-functions, Forum Math. 14 (2002), 419-455.

[10] - - - The pair correlation of zeros of the Riemann zeta-function and distribution of primes, Arch. Math. (Basel) 76 (2001), 41-50.

[11] H. L. Montgomery, The pair correlation of zeros of the zeta function, in: Analytic Number Theory (St. Louis, MO, 1972), Proc. Sympos. Pure Math. 24, Amer. Math. Soc., Providence, 1973, 181-193.

[12] Z. Rudnick and P. Sarnak, Zeros of principal L-functions and random matrix theory, Duke Math. J. 81 (1996), 269-322.

[13] P. Sarnak, L-functions, in: Proc. Internat. Congress of Mathematicians (Berlin, 1998), Doc. Math. 1998, Extra Vol. I, 453-465.

[14] — Letter to Jianya Liu and Yangbo Ye, December 17, 2001.

Department of Mathematics

Shandong University

Jinan, Shandong 250100, China

E-mail: jyliu@sdu.edu.cn
Department of Mathematics The University of Iowa Iowa City, Iowa 52242-1419, U.S.A. E-mail: yey@math.uiowa.edu

Received on 28.10.2003

and in revised form on 12.3.2005 